\title{
Aspectos legales sobre el libro electrónico en México y España
}

Legal aspects regarding the e-books in Spain and Mexico

\author{
Juan Miguel Sánchez Vigil (1), Juan Carlos Marcos Recio (2), Ricardo Villegas TovaR (3), María \\ Olivera ZaLduA (4)
}

(1) Departamento de Biblioteconomía y Documentación. Facultad de Documentación de la UCM. C/ Santísima Trinidad, 37. 28010. Madrid. jmvigil@terra.es. (2) Sección Departamental de Biblioteconomía y Documentación. Facultad de Ciencias de la Información. Adv. Ciudad Universitaria S/N. 28040. Madrid jmarcos@ccinf.ucm.es. (3) Biblioteca "Niels Bohr". Benemérita Universidad Autónoma de Puebla. Edificio 129 Ciudad Universitaria Col. Jardines de San Manuel. Puebla (México) ricardo.villegas@dgb.buap.mx. (4) María Olivera Zaldua. Ateneo de Madrid. molizal@hotmail.com

\begin{abstract}
Resumen
Análisis de los aspectos legales sobre el libro electrónico en España y México. Se realiza un estudio comparativo tomando como referencia aspectos económicos, producción editorial, las leyes sobre el libro de ambos países, los derechos de autor y la propiedad intelectual. Se valoran los contratos de edición y el vacío jurídico en este aspecto. Se contemplan asimismo los problemas de la difusión no autorizada y las medidas de protección al respecto.
\end{abstract}

Palabras clave: Contrato de edición. Derechos de autor. Libros electrónicos. Ley de fomento para la lectura y el libro (México. 23.07.2008). Ley de lectura, del libro y de las bibliotecas (España. 10.07.2007). Propiedad Intelectual.

\section{Introducción}

Es objeto de este trabajo (1) analizar los aspectos legales en relación con el libro electrónico en España y México, señalando la problemática y las medidas emprendidas para sentar las bases de actuaciones futuras. La Association of American Publisher define el libro electrónico como la obra literaria bajo la forma de objeto digital, compuesta de metadatos, destinada a ser publicada y a la que se accede mediante sistema electrónico (2000 p. 56) y Luis Codina lo presenta como (2000 p. 95).

Publicación digital no periódica, es decir, que se completa en un solo volumen o en un número predeterminado de volúmenes, y que puede contener cualquier morfología de información.

Desde el nacimiento de la industria editorial moderna a mediados del siglo XIX hasta la aparición del libro electrónico, las ciencias jurídicas han acompañado a los procesos de producción de obras para tutelar, desde diferentes ramas especializadas, cada uno de los pasos que permiten la existencia de tan valiosos vehículos de cultura. Ya sea con leyes en materia de derecho de autor, leyes que exigen el depósito de

\begin{abstract}
An analysis of the legal aspects concerning the ebooks in Spain and Mexico. A comparative study is carried regarding the financial aspects, the editorial production, the laws on books existing in both countries, author's rights and intellectual property rights. The publishing contracts and the juridical vacuum in regard to this matter are studied. The problems regarding the unauthorized dissemination of e-books and the protective measures that may be adopted in regard to this problem are also discussed.
\end{abstract}

Keywords: Publishing contract. Author's rights. Mexico. Publishing. Spain. Law for the promotion of reading and books (Mexico. 23.07.2008). Law on Reading, Books and Libraries (Spain. 10.07.2007). Electronic books. E-books. Intellectual property.

obras para su conservación y custodia en bibliotecas nacionales, o leyes que regulan el comercio internacional de los libros, el derecho ha cumplido en mayor o menor medida su función de establecer los alineamientos para regular las relaciones de los agentes involucrados en estos procesos.

Ejemplo de la importancia que da el derecho a este tema lo encontramos en la existencia de una variedad de tratados y convenciones de carácter internacional, surgidos al amparo de instituciones internacionales, que desde diferentes ángulos atienden los elementos relativos a la producción y consulta de obras literarias. Es el caso de la Declaración Universal de Derechos Humanos y su referencia al derecho a la educación, información y propiedad intelectual, la Organización de las Naciones Unidas para la Educación, la Ciencia y la Cultura (UNESCO), que trata el libro como objeto cultural, la Organización Mundial de la Propiedad Intelectual (OMPI) que tutela la expresión original contenida en los libros, e incluso la Organización Mundial del Comercio $(\mathrm{OMC})$ con respecto a la enajenación internacional de los bienes intelectuales. Todas ellas han conceptualizado a la obra literaria y a 
su soporte material como bienes sujetos a una protección jurídica internacional, y en ese sentido los países que se adhieran a los respectivos tratados contenidos deben hacer valer sus mandatos de manera directa o realizar las adecuaciones legislativas nacionales necesarias para cumplir con las indicaciones acordadas.

Los problemas acuciantes son la piratería y la reproducción ilícita, que influyen en la econo-mía. Para conseguir eficiencia de las herramientas legales es esencial la política pública de las autoridades, mejorar las leyes, reducir los tiempos de solución de casos en los tribunales. El libro electrónico, por su naturaleza digital, puede ser controlado en cuanto a su transmisión ilegal mediante medidas tecnológicas de protección (TPM) y herramientas como los Digital Rights Management (DRM). Así, autores y editores tienen en el libro electrónico una oportunidad para innovar en el negocio, puesto que los lectores y usuarios están a la expectativa. Los autores apenas han visto remunerado su trabajo digital y son reticentes a permitir versiones digitales que se copian con más facilidad y que apenas les aporta beneficios. Sus derechos han de ser reconocidos y respetados por los editores y por los lectores para que puedan seguir creando.

En consecuencia se crean leyes de alcance nacional que contemplan la lectura y el libro desde ordenamientos que promueven la eliminación o reducción de impuestos y aranceles por la compra o importación del libro, que establecen precios únicos o que protegen contra la ilegalidad. Estas leyes coinciden en una filosofía común: resguardar jurídicamente al libro y sus contenidos como elementos esenciales que coadyuvan a cumplir los objetivos de la educación y el desarrollo social.

El impacto del libro electrónico en la sociedad del conocimiento ha obligado a las instituciones competentes a tomar decisiones de urgencia que no siempre responden a las necesidades o a las situaciones que se plantean, debido a los constantes cambios en el proceso de reconversión. El libro digital plantea numerosas cuestiones, desde la producción, pasando por los lectores, hasta los derechos de autor y la copia ilegal.

El marco legal de la explotación de libros electrónicos tiene como soporte dos tratados internacionales que definen un nuevo concepto denominado derecho de puesta a disposición. Ambos fueron desarrollados por la OMPI en 1996: Tratado sobre Derechos de Autor y Tratado sobre Interpretaciones o Ejecuciones o Fonogramas. De ambos se deriva una directiva de la Comunidad Europa (2001/129/CE) en la que se incluye el derecho de puesta a disposición dentro de comunicación pública, y en la que se explica:

Los estados miembros establecerán a favor de los autores el derecho exclusivo a autorizar o prohibir cualquier comunicación al público de sus obras, por procedimientos alámbricos e inalámbricos, incluida la puesta a disposición del público de sus obras de tal forma que cualquier persona pueda acceder a ellas desde el lugar y el momento que elija.

\section{Producción de libros electrónicos en España y México}

La evolución de la industria editorial viene dada por la globalización, la divulgación de las nuevas tecnologías, la difusión y explotación a través de Internet y la subordinación del sector a la red de comunicación (Sánchez Vigil, 2009 p.12). El crecimiento de las publicaciones digitales es evidente, pero no con la fuerza que los expertos auguraban a comienzos del siglo XXI, confirmado por los datos del ministerio de Cultura español en el informe sobre el impacto del libro electrónico del sector editorial (2010), que indican un crecimiento del $48 \%$ en 2009 respecto a 2008. Visto así, el avance parece imparable, pero las cantidades son mínimas al partir de una producción reducida en comparación con los libros impresos. Este mismo informe indica que la digitalización de contenidos apenas alcanza el $5 \%$ de la producción de las empresas, si bien la previsión a corto plazo es alcanzar una media entre el 50 y el $100 \%$ de la producción.

\begin{tabular}{lll}
\hline Editoriales & $\begin{array}{l}\text { Catálogo } \\
2009\end{array}$ & $\begin{array}{l}\text { Previsión } \\
\text { para 2011 }\end{array}$ \\
\hline $\begin{array}{l}\text { Grandes } \\
5.000-10.000 \text { obras }\end{array}$ & $5 \%$ & $50-100 \%$ \\
\hline $\begin{array}{l}\text { Medianas } \\
2.500-5.000 \text { obras }\end{array}$ & $5 \%$ & $50-100 \%$ \\
\hline $\begin{array}{l}\text { Pequeñas } \\
500-1.000 \text { obras }\end{array}$ & $5 \%$ & $50-100 \%$ \\
\hline
\end{tabular}

Tabla I. Impacto de la digitalización en el catálogo de las editoriales españolas

Los datos de la Cámara Nacional de la Industria Editorial Mexicana, encargada de la recopilación de datos sobre la materia, indican que en la primera década del siglo $\mathrm{XXI}$ la producción de libros aumentó en más de un 40\%, pasando de 14.726 en 2004 títulos a 20.698 en 2007. De los 229 editores privados que se encontraban censados en 2007, sólo 12 pertenecían a grandes compañías, lo que significa una gran concentración del producto. En los años anteriores, sin embargo, se había producido una recesión, pasando de 16.000 ejemplares en 2000 a 15.542 en 2002. En esos mismos años la edi- 
ción española no experimentó apenas aumento, por el exceso de títulos en el mercado.

\begin{tabular}{lrrr}
\hline & Total & Impresos & Otros soportes \\
\hline 2003 & 82.196 & 77.950 & 4.246 \\
\hline 2004 & 82.207 & 77.376 & 4.831 \\
\hline 2005 & 81.312 & 76.265 & 5.047 \\
\hline 2006 & 81.451 & 77.330 & 4.121 \\
\hline 2007 & 82.559 & 75.006 & 7.553 \\
\hline 2008 & 88.330 & 80.229 & 6.101 \\
\hline 2009 & 96.460 & 83.210 & 13.250 \\
\hline TOTAL & 592.515 & 547.366 & 45.149 \\
\hline
\end{tabular}

Tabla II. Producción editorial en España, 2003-2009 (Panorámica de la Edición en España, Ministerio de Cultura)

\begin{tabular}{rrrr}
\hline & Total & Impresos & \multicolumn{2}{c}{ Otros soportes } \\
\hline 2003 & 15.571 & 15.233 & 338 \\
\hline 2004 & 15.197 & 14.726 & 471 \\
\hline 2005 & 18.741 & 18.310 & 431 \\
\hline 2006 & 19.102 & 18.682 & 420 \\
\hline 2007 & 20.698 & 20.300 & 398 \\
\hline 2008 & 20.568 & 20.252 & 316 \\
\hline 2009 & - & - & - \\
\hline Total & 109.877 & 107.493 & 2.384 \\
\hline
\end{tabular}

Tabla III. Producción editorial en México, 2000-2009 (Cámara Nacional de la Industria Editorial Mexicana -CANIEM-)

\begin{tabular}{rcccrr}
\hline & Electrónica & $\begin{array}{c}\text { Micro } \\
\text { formas }\end{array}$ & $\begin{array}{r}\text { Video } \\
\text { libros }\end{array}$ & $\begin{array}{r}\text { Audio } \\
\text { libros }\end{array}$ & Total \\
\hline 2003 & 209 & - & - & 139 & 338 \\
\hline 2004 & 453 & - & - & 18 & 471 \\
\hline 2005 & 416 & - & - & 15 & 431 \\
\hline 2006 & 409 & - & - & 11 & 420 \\
\hline 2007 & 394 & - & - & 4 & 398 \\
\hline 2008 & 307 & - & - & 9 & 316 \\
\hline 2009 & - & - & - & - & - \\
\hline Total & 2.188 & - & - & 196 & 2.384 \\
\hline
\end{tabular}

Tabla IV. Producción editorial en soportes no impresos en México (Cámara Nacional de la Industria Editorial Mexicana -CANIEM-)

\section{Las leyes del libro en España y México}

Las leyes sobre el libro en España y México se aprobaron con un año de diferencia, la primera en junio de 2007, la segunda en julio de 2008. Sus objetivos muestran similitudes en los aspectos esenciales como la difusión del libro, la salvaguarda de la lectura y el apoyo a las nuevas tecnologías como medio de conformación de una sociedad culta, participativa y dinámica dentro de la sociedad del conocimiento.

3.1. Ley de lectura, del libro y de las bibliotecas (España, 2007)

España ha participado activamente en la firma de instrumentos que promuevan la lectura, el libro y todos los procesos editoriales involucrados. Igualmente ha realizado iniciativas para ofrecer soluciones legislativas. El artículo 44 de la Constitución se refiere a la importancia de resguardar desde el Estado el derecho a la cultura, desarrollado después en la Ley del libro, la lectura y las bibliotecas cuyo objeto precisamente es:

Definir el marco jurídico del libro, en atención a su carácter de producto cultural, desde su creación hasta su comercialización, difusión y conservación como parte del patrimonio bibliográfico español; de las publicaciones seriadas, del fomento de la lectura, de las bibliotecas y, en especial, de la cooperación bibliotecaria. Es de aplicación al libro, en cuanto a su edición y comercialización en cualquier tipo de soporte susceptible de lectura y a las publicaciones seriadas. Es también de aplicación al fomento de la lectura y del hábito lector y a las bibliotecas, de acuerdo con el marco competencial establecido en la Constitución y en los respectivos estatutos de autonomía.

El concepto de libro es interesante en cuanto que comprende los impresos, los electrónicos e incluso los contenidos en otros soportes aún no conocidos:

Obra científica, artística, literaria o de cualquier otra índole que constituye una publicación unitaria en uno o varios volúmenes y que puede aparecer impresa o en cualquier otro soporte susceptible de lectura. Se entienden incluidos en la definición de libro, a los efectos de esta Ley, los libros electrónicos y los libros que se publiquen o se difundan por Internet o en otro soporte que pueda aparecer en el futuro, los materiales complementarios de carácter impreso, visual, audiovisual o sonoro que sean editados conjuntamente con el libro y que participen del carácter unitario del mismo, así como cualquier otra manifestación editorial.

La Ley del Libro lo tutela en todas sus acepciones desde una doble apreciación: como elemento cultural y como bien económico en el mercado. Para ello identifica todos los procesos de producción y comercialización. Para lograr estas encomiendas establece las reglas y sanciones adecuadas, manteniendo el precio único del libro y permitiendo que el consumidor, independientemente de su ubicación geográfica, acceda al material en condiciones igualitarias de precio. 
3.2. Ley de fomento para la lectura y el libro (México, 2008)

El artículo cuarto (p. 9) de la Constitución mexicana, al igual que en el caso español, establece el derecho de acceso a la cultura y la diversidad de su expresión en función de sus creadores como un medio de entendimiento y participación social en el quehacer nacional. La interpretación de este artículo conduce a la Ley de fomento para la lectura y el libro promulgada en 2008 con el fin establecer las condiciones jurídicas de tutela a las actividades que se han englobado bajo el concepto "cadena del libro". Así incluye en su espectro de protección al conjunto de actividades realizadas por las personas que inciden en la creación, producción, distribución, promoción, venta y lectura del libro. Su objeto es pues:

Propiciar la generación de políticas, programas, proyectos y acciones dirigidas al fomento y promoción de la lectura; fomentar y estimular la edición, distribución y comercialización del libro y las publicaciones periódicas; fomentar y apoyar el establecimiento y desarrollo de librerías, bibliotecas y otros espacios públicos y privados para la lectura y difusión del libro; establecer mecanismos de coordinación interinstitucional con los distintos órdenes de gobierno y la vinculación con los sectores social y privado, para impulsar las actividades relacionadas con la función educativa y cultural del fomento a la lectura y el libro; hacer accesible el libro en igualdad de condiciones en todo el territorio nacional para aumentar su disponibilidad y acercarlo al lector; fortalecer la cadena del libro con el fin de promover la producción editorial mexicana para cumplir los requerimientos culturales y educativos del país; estimular la competitividad del libro mexicano y de las publicaciones periódicas en el terreno internacional, y estimular la capacitación y formación profesional de los diferentes actores de la cadena del libro y promotores de la lectura.

El libro se convierte en el objeto de la ley y se define en su artículo segundo con la siguiente declaración:

Toda publicación unitaria, no periódica, de carácter literario, artístico, científico, técnico, educativo, informativo o recreativo, impresa en cualquier soporte, cuya edición se haga en su totalidad de una sola vez en un volumen o a intervalos en varios volúmenes o fascículos. Comprenderá también los materiales complementarios en cualquier tipo de soporte, incluido el electrónico, que conformen, conjuntamente con el libro, un todo unitario que no pueda comercializarse separadamente.

En esta definición se observa una omisión semántica en referencia al libro electrónico, que se compensa al incluir por equivalencia al mencionar la frase "impreso en cualquier soporte". Por tanto, el libro impreso y el electrónico que- dan ampliamente considerados como elementos base para el fomento de la lectura.

Al igual que en España la Ley del libro de México exige la intervención de ciertas autoridades, e inclusive crea algunas, para que en virtud de sus funciones generen políticas encaminadas al estimulo de la actividad editora, el establecimiento de librerías y bibliotecas y permite el acceso al libro en condiciones igualitarias de precio con independencia del lugar geográfico donde se adquiera el libro.

\subsection{Análisis comparativo}

De esta forma nace el concepto de "libro" desde la perspectiva del derecho de autor. Para el caso mexicano éste se define en la Ley Federal de Derechos de Autor (LFDA) artículo 123 a partir de la transcripción literal que se hace del contenido del citado artículo 2 de la Ley de fomento para la lectura y el libro (México) pero que, como ya se ha comentado, no incluye expresamente el concepto "libro electrónico" como así lo hace la Ley del Libro de España en su artículo 2.

La Ley de Propiedad Intelectual de España no dedica un artículo para definir lo que es un libro, pero esta aparente ausencia queda plenamente resarcida por el citado artículo 2 de la Ley del Libro española que lo define en abundancia hasta el punto de incluir lo relativo a su versión electrónica. La protección legal que se da al libro electrónico en México desde el derecho de autor se ofrece por analogía, no tanto por su soporte, sino por su contenido reconocido como obra literaria. Por su parte la Ley de Propiedad Intelectual española tampoco utiliza el concepto "libro electrónico" pero igualmente lo protege por considerarlo como una obra literaria que se soporta en un medio tangible o intangible, conocido o por conocerse.

Ahora bien, el libro impreso no solo cuenta con su volumen físico para expresar sus contenidos, también se puede hacer acompañar de otro tipo de soportes, incluido el electrónico. Para esos casos, tanto la LFDA como la Ley de Propiedad Intelectual (LPI) incluyen expresamente la protección que se debe hacer a esos elementos complementarios que no deben confundirse con el libro electrónico cuya existencia es completamente independiente de una posible versión impresa.

Por otra parte, la Ley del libro española, en el artículo 7 (Promoción de la industria editorial y del comercio del libro), declara el apoyo institucional al fomento del hábito del lector y de las 
tecnologías aplicadas a la gestión e intercambio de información:

La Administración General del Estado y sus organismos públicos colaborarán con las asociaciones de profesionales del sector del libro español en todas aquellas actividades relacionadas con el fomento del hábito lector y de la difusión del libro, en aquellas que propicien una mejor organización profesional y en el desarrollo de servicios que puedan repercutir en beneficio de los lectores o del comercio del libro, así como en el fomento de las tecnologías aplicadas a la gestión, los intercambios de información y la formación. Dicha actividad deberá realizarse en colaboración y cooperación con las comunidades autónomas cuando afecte a entidades o actividades que se realicen en su territorio.

Una de la diferencias entre las dos leyes es el capítulo de sanciones, excluido de la mexicana e incluido en la española desde el capítulo 16 en adelante, especificando el régimen sancionador por incumpliendo de la Ley, indicando las multas por los delitos cometidos.

\section{Propiedad intelectual y derechos de autor}

La cesión por la vía contractual de los derechos de explotación del autor a favor del editor es el acto jurídico que da inicio al proceso de producción del libro. De ahí que las legislaciones dediquen capítulos a regular las condiciones generales que deben preservarse en el momento de cesión y que por su ejercicio deberán traducirse en beneficios económicos, tanto para el autor como para el editor.

Propiedad intelectual y derechos de autor son términos que convergen en cuanto a su finalidad. En 2003 se celebró en México el Primer Foro de Expertos de Derechos de Autor en edición electrónica organizado por el Fondo de Cultura Económica, del que resultaron una serie de recomendaciones aplicables a los contratos de edición: necesidad de un esquema de contratación ágil y confiable, respeto a los principios básicos del derecho de autor para estructurar los contratos en edición electrónica, participación del autor en la explotación de la obra y desarrollo de sistemas para la gestión electrónica de derechos (Sánchez Vigil, 2009 p. 283).

En España, la propiedad intelectual se encarga de la protección de las obras literarias, artísticas o científicas en virtud de la originalidad con la que sean expresadas y siempre que se encuentren soportadas en un bien tangible o intangible, conocido o por conocer. Así es como lo establece el artículo 10 del Texto Refundido de la Ley de Propiedad Intelectual. En México, la rama jurídica que protege las obras literarias y artísticas recibe el nombre de derecho de autor y sus estatutos están inscritos en la ley que lleva ese mismo nombre: Ley Federal del Derecho de Autor.

La transmisión de los derechos patrimoniales del autor a favor del editor va a dar origen al nacimiento de nuevos derechos y obligaciones que se enmarcarán por los acuerdos a los que lleguen las partes en el contrato de edición que celebren. Éste contrato se encuentra reglamentado a partir del artículo 58 de la LPI (España) y para el caso de México en el 42 de la LFDA. Ambos coinciden en indicar la obligación que tiene el autor de entregar la obra literaria terminada al editor para que éste la reproduzca y distribuya con fines comerciales. Para que el editor pueda cumplir con su parte del acuerdo tendrá que realizar una serie de acciones que conviertan los originales del autor en un libro de interés para el interesado.

Una y otra ley definen un objeto de protección: la obra literaria, científica o artística, y al mismo tiempo se refieren al creador de la obra, persona que realiza los esfuerzos intelectuales y expone su dominio de la técnica para expresar en ella sus conocimientos; es decir, quien imprime su impronta a la obra. Las dos leyes otorgan al autor derechos personales o morales y derechos patrimoniales o de explotación económica. En realidad no se trata de dos derechos, sino de dos aspectos del mismo derecho de autor. Los derechos personales se enfocan a las facultades que tiene el creador para decidir si ha de divulgar su obra, si la ha de modificar o si la ha de retirar del comercio. Asimismo este derecho moral asiste al creador para exigir la autoría y mantener la integridad de la obra.

Los derechos personales son irrenunciables e inalienables, no así los de explotación que tienen vigencia temporal: en el caso de España hasta setenta años después de la muerte del autor y en el mexicano hasta cien. Se plantea aquí una cuestión de interés sobre la comparación de plazos, aclarada en el artículo 7 (párrafo 8) del Convenio de Berna para la Protección de las Obras Literarias y Artísticas, y del que España y México son partes firmantes. La respuesta es clara, ya que el hecho de que en México la obra se proteja más tiempo que en España no quiere decir que la obra exportada a España sea protegida por cien años, sino que se respetaría el tiempo que corresponde en virtud de lo que se estipule para las obras españolas. De lo contrario se produciría una afectación de los derechos de autores españoles en su propio territorio. En sentido contrario, la obra española exportada a México sí tendrá una protección posterior a la muerte del autor por un lapso de cien años en virtud de ejercer el principio de trato nacional. 


\subsection{El contrato de edición de los libros} electrónicos

Los autores están de acuerdo en lo que respecta a la insuficiencia del marco jurídico en el tema de los contratos. En Estados Unidos existen ya dos tipos de contratos, uno para formato papel y otro para formato digital (Celaya, 2007), mientras que en Europa, la ley francesa denominada Hadopi pretende sancionar la descarga ilegal de las obras intelectuales a partir de controlar la transmisión de datos por Internet.

La legislación española no está definida, y como señala Sánchez Almeida (2010) los artículos 58 y siguientes de la Ley de Propiedad Intelectual española se redactaron pensando en la reproducción y distribución de obras en formato físico, y especialmente la edición en forma de libro. El informe del Observatorio de la Lectura y el Libro (2010) del Ministerio de Cultura especifica que:

\begin{abstract}
Cabe pensar que contratos de edición antiguos no harán referencia al derecho de puesta a disposición esencial para la descarga de libros electrónicos en Internet, bien por no existir en el momento de la firma este derecho tal y como esta configurado ahora en el ordenamiento jurídico, o bien por no ser Internet el medio habitual de explotación o por otras razones similares.
\end{abstract}

La controversia es significativa: mientras quw para los representantes de los autores del libro digital se deben realizar contratos específicos, algunas voces autorizadas no están convencidas de ello. Así, Antonio María Ávila (Muñoz y Rodríguez 2010 p. 43), director de la Federación de Gremios de Editores de España, plantea que

todavía no está claro si el e-book debe tratarse como contrato de edición o como comunicación pública. Si es lo primero, caduca en diez años, si lo segundo, puede ser ilimitado.

La falta de definición legal obliga a reelaborar los contratos para los formatos electrónicos, y así lo indica la Asociación Colegial de Escritores Españoles en sus recomendaciones (2010), manifestando que deben realizarse contratos diferentes para libro electrónico, móvil u otro formato, indicando el soporte de difusión, el tiempo de cesión, el sistema de control de descargas o ventas, las formas de liquidación en caso de anticipos o descargas, la separación de los libros ya editados por sistemas tradicionales (derechos por ventas) y libros nuevos (derechos por descargas). La Asociación Colegial de Escritores Españoles entiende que la cesión digital no es un contrato de edición, sino una licencia de explotación. Ello supone la realización de un acuerdo para cada caso (cesión), lo que significa que deben considerarse no válidas las cláusulas de los contratos de libros impresos.
La controversia es obvia, si bien algunos editores tienen ya despejada la incógnita, entre ellos Antonio Quirós, consejero de la editorial Luarna.com (J. A. Muñoz: 2010):

El derecho de explotación digital es un derecho independiente al de explotación impresa. Todos los autores que tienen libros publicados en papel son propietarios de su derecho digital y pueden hacer con él lo que quieran. Pero las editoriales tradicionales, en estos dos últimos años han seguido un camino curioso, primero recomendando a sus autores que no negocien dicho derecho con terceros (aunque dicho sea de paso ellos tampoco han estado dispuestos a editar en digital) y en los contratos nuevos reservándose dicho derecho (cosa legalmente más que dudosa) aunque sin ejercerlo de hecho. Así, pues, un autor en sus contratos antiguos se encuentra que no tiene cedido el derecho digital, pero su editorial no le deja cederlo y en los nuevos se lo reserva ya por si acaso.

\subsection{El problema de la difusión no autorizada} ilegal y las medidas de protección

El tema de la reproducción ilícita o difusión no autorizada es ciertamente grave. La Coalición de Contenidos Culturales y el Centro Español de Derechos Reprográficos (CEDRO) detectó en 2010 más de doscientas webs dedicadas a la piratería digital de libros. La respuesta en algunos países ha sido la elaboración de leyes específicas, como el caso de Francia con la llamada ley Hadopi para la lucha contra las descargas ilegales por Internet.

El informe Percepción sobre el clima empresarial editorial y tendencias a corto plazo (CERLAC, 2009), señala que las principales amenazas a la industria del libro en Latinoamérica no radican exclusivamente en la piratería y en el fotocopiado ilegal, sino en la alteración en las variables macroeconómicas y la baja demanda del producto. Por su parte, la Alianza Internacional de la Propiedad Intelectual (IIPA) indica en el informe 301 de febrero de 2010 que en México el fotocopiado de libros es un grave conflicto y que es urgente la implementación de políticas de control. La respuesta al problema la ha dado en parte el Centro Mexicano de Protección y Fomento de los Derechos de Autor (CEMPRO) que ha optado, como en el caso español de CEDRO, por la concesión de licencias globales a las instituciones y empresas implicadas.

El problema español sobre la copia ilegal no se encuentra en el fotocopiado sino en el elevado tráfico ilícito de obras por Internet, específicamente en las redes $\mathrm{P} 2 \mathrm{P}$, lo que se traduce en las reservas de los editores sobre la viabilidad del negocio del libro electrónico en las condiciones actuales. En la encuesta de la Federación 
del Gremio de Editores realizada en febrero de 2010 sobre 254 empresas la oferta de versiones electrónicas de obras en los catálogos es muy reducida, ya que apenas supone el $5 \%$ del total, y en ello influye sin duda el miedo a la piratería.

Entre las medidas de protección de los libros electrónicos, una vez que han sido preparados para su difusión en la red, se encuentran los Digital Rights Management (DMRs), que controlan totalmente el acceso o bien lo limitan e informan sobre las condiciones en que deben gestionarse los derechos. Ahora bien, la aplicación de los DRMs supone limitaciones para el consumidor sobre los libros electrónicos y la situación resulta controvertida en el caso de centros públicos, en especial las bibliotecas virtuales que podrían encontrarse con trabas a la difusión de contenidos. Además de las medidas tecnológicas como los DRMs, se emplean otras de tipo jurídico como las licencias que regulan el acceso. Entre estas se encuentran las alternativas denominadas Copyleft, basadas en el software libre, y dentro de estas las Creative Commons, pensadas para los contenidos artísticos.

La legislación española contempla la protección desde dos vías: la civil y la penal. La primera regulada por la Ley de Propiedad Intelectual (artículos 138 a 143), y la segunda por la Ley Orgánica del Código Penal 10/1995 (artículos 270-272 y 287-288). Aun así, la desprotección de los titulares de los derechos es evidente y por ello las instituciones públicas reconocen la necesidad de readaptar el marco legal a la nueva realidad, es decir que se hace imprescindible su revisión para responder a la difusión no autorizada. Una respuesta ha sido la bajada de los precios a través de la reducción del IVA o impuesto añadido en determinados productos electrónicos, con el fin de equipararlos a los libros impresos (4\%), pero el ministerio de Hacienda ha aclarado que sólo los libros electrónicos que se vendan en soporte físico (CD-Rom, pendrive o USB) podrán acogerse al tipo reducido, mientras que el resto seguirá el tipo general (18\% desde julio de 2010). La razón es que este modelo de transacciones se considera comercio electrónico y por tanto debe aplicarse el artículo 98.2 de la directiva europea sobre servicios electrónicos (2006).

\section{Conclusiones}

1. El marco jurídico internacional y nacional que tutela los contenidos de los libros electrónicos es amplio, si bien pendiente de una reforma condicionada por la evolución constante. En general, los principios están definidos y los cambios dependerán de la producción de libros electrónicos y de los planteamientos de los editores.

2. La producción editorial en España es cuatro veces superior a la mexicana, ello sin tener en cuenta que la población de México es más del doble que la de España.

3. La producción en soportes digitales es muy reducida en México (316 títulos en 2008). Mientras que en España se observa un constante crecimiento (100\% entre 2008 y 2009), en México la edición digital se encuentra estancada.

4. Ambos países cuentan con nueva legislación sobre el libro, aprobada en 2007 (España) y 2008 (México), con un marco jurídico similar, excepto en materia de sanciones por incumplimiento.

5. Aspecto significativo es la vigencia temporal de los derechos de autor (70 años para España y 100 para México), lo que provoca problemas jurídicos.

\section{Notas}

(1) Este trabajo se ha realizado en el marco del proyecto CSO2009-0761 financiado por el Ministerio de Ciencia y Tecnología.

\section{Referencias}

Administración electrónica y el servicio a los ciudadanos: el Ministerio de Economía y Hacienda ante los retos de la Ley 11/2007, de Acceso Electrónico de los Ciudadanos a los Servicios Públicos, La (2009). Madrid: Ministerio de Economía y Hacienda.

Alonso Pereda, Elena (2004). El sector editorial en México. ICEX. Oficina Económica y Comercial de la Embajada de España en México.

Association of American Publisher (2000). Metadata Satandards for Ebooks. Version 1.0. Nueva Cork, 2000.

Celaya, Javier (2007). Tendencias Web 2.0 en el sector del libro digital. Dosdoce.com. http://www.dosdoce.com /continguts/ (2010-04-15).

Codina, Luis (2000). El libro digital y la www. Madrid: Tauro

Debaten editores ley del libro (2008). // Reforma. http:www.presidencia.gob.mx/prensa/ (2010-02-26).

Elosua, Marcelino (2009). Informe Omniprom 2009 sobre el libro en México. México: LID www.omniprom.com.

Encuesta sobre el libro digital: Impacto de la digitalización en el catálogo, canales de distribución y de venta y política de precios. http://www.dilve.es/ (2010-04-11). Federación de Gremios de Editores de España (2010).

Fernández Abad, Francisco Javier (2007). El uso beneficioso del libro impreso y del libro digital // Documentación de las Ciencias de la Información. 30 277-294.

Franganillo, Jorge (2008). "La industria editorial frente al libro electrónico" // El Profesional de la Información.17 416-417.

García Marco, Francisco Javier (2008). El libro electrónico y digital en la ecología informacional: avances y retos // El Profesional de la Información. 17 373-389. 
Haute autorité pour la diffusion des œuvres et la protection des droits sur Internet (Francia, 2009).

Informe 2009 de la industria de contenidos digitales. Madrid: ASIMELEC, Asociación Multisectorial de Empresas de Tecnologías de la Información, Comunicaciones y Electrónica.

International Intellectual Property Alliance (2010). Country Reports, $\mathrm{n}^{\circ} 301 \mathrm{http}: / / \mathrm{www}$.iipa.com/countryreports.html (2010-4-11).

Larrañaga Rubio, Julio (2008). El mercado del e-book en España: un análisis económico // Documentación de las Ciencias de la Información. 31 183-209.

Ley Federal del Derecho de Autor (México, 2003).

Ley de Patentes de Invención y Modelos de Utilidad (España, 1986).

Ley de la Propiedad Industrial (México, 2010).

Ley de Propiedad Intelectual. Texto refundido (España, 1996).

Libro electrónico, El. Ministerio de Cultura. http://www. mcu.es/libro/docs/2010. (2010-04-12)

López Maza, Sebastián (2009). Límites del Derecho de reproducción en el entorno digital. Granada: Comares.

López Suárez, Mercedes; Larrañaga Rubio, Julio. El e-book: Aspectos culturales y socioeconómicos del sistema editorial online // Documentación de las Ciencias de la Información. 28 (2005) 257-267.

López-Tarruella Martínez, Aurelio (2008). Litigios transfronterizos sobre derechos de propiedad industrial e intelectual. Madrid: Dykinson.

Macías Castillo, Agustín (2008). El derecho de autor y las nuevas tecnologías: reflexiones sobre la nueva reforma de la ley de propiedad intelectual. Madrid: La Ley.

Millán, José Antonio (2007). El polimorfo libro electrónico // El Profesional de la Información. 17 369-371.

Moreno Martínez, Juan Antonio (2008). Límites a la propiedad intelectual y nuevas tecnologías: incidencias por la Ley 23/2006, de 7 de julio. Madrid: Dykinson.
Muñoz de Solano y Palacios, Bárbara (2004). El libro impreso ¿un clásico desplazado por el libro electrónico? // Documentación de las Ciencias de la Información. 24 303-322.

Ochoa Arribas, Carlos (2008). Principales novedades de la Ley 10/2007, de 22 de junio, de la lectura, del libro y de las bibliotecas // Revista Aranzadi de Derecho y Nuevas Tecnologías. 67-78.

Oghojafor, Kingsley (2005). E-book publishing success: How anyone can write, compile and sell e-books on the Internet. Oxford: Chandos Publishin.

Percepción sobre el clima empresarial editorial y tendencias a corto plazo (2009) // Cerlac, Boletín, 8 (Febrero 2009) // http://www.cerlalc.org/Percepcion_8.pdf (2010-04-10).

Polo, Magda (2009). Las fronteras del libro electrónico: reflexiones desde la edición académica // Trama y Texturas. (Marzo, 2009) 73-88.

Rodríguez, Joaquín (2007). Edición 2.0: Los futuros del libro. Barcelona: Egedsa.

Rodríguez Tapia, José Miguel (2009). Comentarios a la ley de propiedad intelectual. (Texto refundido, R.D.Leg.1/1996, de 12 de abril). Madrid: ThomsonCívitas.

Sánchez Almeida, Carlos (2010). El mecanoscrito de Averroes. Libro electrónico y derechos de autor // http:// www.kriptopolis.org/libro-electronico-ebook-derechos-de -autor (2010-03-25)

Sánchez Vigil, Juan Miguel (2009). La edición en España. Gijón: Trea.

Subías, Carlos; Angueral, Juame; Vicent, Lluis. Aproximación a la teoría de antenas mediante un libro electrónico interactivo. http://e-spacio.uned.es/fez/eserv.php?pid= taee:congreso-2004-1061\&dsID=S2E02.pdf.

Tratado de la Organización Mundial de la Propiedad Intelectual sobre Derecho de Autor (OMPI, 1996).

Recibido: 13-05-2010. Revisado: 08-07-010.

Aceptado: 08-07-2010. 